\title{
Sugammadex reversal of inadvertent subcutaneous injection of rocuronium - a case report
}

\author{
Anderson César de Oliveira, Vitor Gonçalves Savoia, Ana Cristina P. Mendes Pereira \\ Department of Anaesthesiology, National Cancer Institute - Rio de Janeiro, Brazil
}

\begin{abstract}
Background
It is estimated that drug-related errors occur in one out of five doses given to patients in hospitals ${ }^{1}$, clearly preventable events that often result in serious morbidity for patients and legal disputes for health care professionals. In a review from the ASA Closed Claims Project, immediate and major physiologic effects happened in $47 \%$ of administration errors, $24 \%$ resulted in death and $34 \%$ in serious, long-lasting or permanent injury ${ }^{2}$. In another series ${ }^{3}$, the most common error was a syringe swap, and usually involved a muscle relaxant.

Supportive care is normally the mainstay of treatment, nonetheless, specific reversal of the offending agent is sometimes available and proves to be a valuable ally. Situations in which the error comprises equivocal routes of administration add to the pharmacokinetic complexity of the treatment.

Here we present a case of inadvertent administration of rocuronium. This case is unique as it is the first in the literature we have knowledge of in which the injection was given subcutaneously.
\end{abstract}

\section{Case report}

A $62 \mathrm{~kg}, 78$ year-old female patient was scheduled for facial reconstructive plastic surgery using skin flaps in our tertiary cancer center. Her past medical history was relevant only for poorly treated hypertension. There was no history of allergies or prior anaesthetic complications. Upon admission to the OR, she was sedated with dexmedetomidine $(0,5 \mathrm{mcg} / \mathrm{kg} / \mathrm{h})$, intravenous lidocaine $(1,5 \mathrm{mg} / \mathrm{kg})$ and magnesium sulphate $(30 \mathrm{mg} / \mathrm{kg})$, and bilateral supra-trochlear, infra-orbital, mentonian and pre-auricular nerve blocks were associated for analgesia, along with local infiltration of the field by the surgeon. A total of $10 \mathrm{ml}$ of $2 \%$ lidocaine was used, prepared just before the patient's arrival by the anaesthetics resident.

The patient tolerated the procedure under conscious sedation, but within fifteen minutes of peripheral nerve block onset she became tachycardic and hypertensive, showed signs of increased respiratory work and desaturation in room air. Initial loading doses were immediately stopped and she was intubated for airway protection. The procedure was completed under general anaesthesia with propofol, suxamethonium and sevofluorane. Mislabelling of the local anaesthetic syringe was presumed after an empty vial of rocuronium was found onto the medication cart where the syringes had been prepared. She was then treated with $400 \mathrm{mg}$ of sugammadex, with complete reversal of symptoms. Neuromuscular monitoring was arranged, and revealed a T4 / T1 ratio of $>90 \%$ in a train of four test around fifteen minutes after the reversal attempt. The patient was uneventfully extubated and kept under close surveillance at the PACU. She did not exhibit any further signs of muscular fatigue or dyspnea during four hours of observation, after which she was discharged to the ward.

\section{Discussion}

Sugammadex is a modified $\gamma$-cyclodextrin that irreversibly binds rocuronium, and we report a case of its use in the reversal of inadvertent neuromuscular blockade provoked by a subcutaneous injection. This is the first case reported to our knowledge in which the unexpected administration route became a safety concern, and was successfully managed. Sugammadex appeared to have both a safe and effective pharmacokinetic profile for reversal of rocuronium neuromuscular blockade as a single dose, in spite of the unpredictable pharmacokinetics and erratic absorption by the subcutaneous route. Its short elimination half-life of around two hours ${ }^{3}$ could become a concern should rocuronium recirculation occur, and more studies are needed to establish it as a mainstay of treatment in every resemblant case and the optimal dosing regimen.

\section{Learning points}

Medication mislabeling is one of the most common causes of anaesthetic misadventures, and results in significant morbidity and mortality. Standardised syringe identification tags should be included in institutional safety protocols.

Health care professionals ought to keep a high index of suspicion when faced with incompatible or unexpected drug reactions, as failure to link those to unduly drug administration might be potentially fatal. Specific drug reversal agents should be used whenever feasible in such circumstances.

In one case, sugammadex proved to be safe and effective for complete reversal of subcutaneously injected rocuronium in spite of its erratic absorption, up to 5 hours after infiltration.

\section{Disclosures}

None.

\section{References}

1. Barker KN et al. Arch Intern Med 2002; 162:1897-1903.

2. Bowdle, TA. ASA Newsletter 2003; 67(6):11-13.

3. Orser BA et al. Can J Anaesth 2001; 48:139-146.

4. Bridion (sugammadex) prescribing information. MSD, UK, 2015. 